\title{
A Family of Discontinuous Galerkin Finite Elements for the Reissner-Mindlin Plate
}

\author{
Douglas N. Arnold, ${ }^{1}$ Franco Brezzi, ${ }^{2}$ and L. Donatella Marini ${ }^{3}$
}

Received October 6, 2003; accepted (in revised form) April 23, 2004

\begin{abstract}
We develop a family of locking-free elements for the Reissner-Mindlin plate using Discontinuous Galerkin (DG) techniques, one for each odd degree, and prove optimal error estimates. A second family uses conforming elements for the rotations and nonconforming elements for the transverse displacement, generalizing the element of Arnold and Falk to higher degree.
\end{abstract}

KEY WORDS: Discontinuous Galerkin; Reissner-Mindlin plates; locking-free finite elements.

\section{INTRODUCTION}

Recently there has been a considerable interest in the development of Discontinuous Galerkin (DG) methods for elliptic problems (see, for instance, [4] and the references therein). Although their practical interest is still under investigation, it is clear that the DG approach often implies a different way of dealing with the problem, that can sometimes lead to new conforming or nonconforming finite elements that would have been more difficult to discover starting with a classical approach. Examples in this direction are, for instance, the extension of the Crouzeix-Raviart element for Stokes problem or nearly incompressible elasticity problems [23], and the recent paper by two of the present authors using DG methods to develop nonconforming elements for the Reissner-Mindlin plate [16].

\footnotetext{
${ }^{1}$ Institute for Mathematics and its Applications, University of Minnesota, Minneapolis, MN 55455. E-mail: arnold@ima.umn.edu

${ }^{2}$ Dipartimento di Matematica, Università di Pavia, Italy and IMATI-CNR, Via Ferrata 1, 27100 Pavia, Italy. E-mail: brezzi@imati.cnr.it

${ }^{3}$ Dipartimento di Matematica, Università di Pavia, Italy and IMATI-CNR, Via Ferrata 1, 27100 Pavia, Italy. E-mail: marini@imati.cnr.it
} 
Here we present a family of finite element approximations for the Reissner-Mindlin plates. These are mixed methods, in which the rotation vector, transverse displacement, and transverse shear are all approximated. The starting point of the family is a totally discontinuous approach, in which both rotations $\boldsymbol{\theta}$ and transversal displacements $w$ are locally polynomials of degree $\leqslant k$, where $k$ is an odd integer, while the transverse shears are approximated by totally discontinuous polynomials of degree $k-1$. However, many variants are possible. For instance, we could (i) keep $\boldsymbol{\theta}$ discontinuous but use a nonconforming $w$ (having moments up to the order $k-1$ continuous at the interelement boundaries), or (ii) take both $\boldsymbol{\theta}$ and $w$ nonconforming (by adding a suitable set of bubble functions to $\theta$ ), or (iii) use a continuous $\boldsymbol{\theta}$ and a nonconforming $w$, by adding a different set of bubble functions to $\boldsymbol{\theta}$. This last option, for $k=1$, will give back the Arnold-Falk (AF) element [5], and therefore, for $k>1$, can be seen as a higher order version of AF. On the other hand, the other options can be seen, for $k=1$, as a discontinuous or nonconforming versions of AF. In particular here we present the analysis of the two extreme cases, that is the fully discontinuous case and the case in which $\boldsymbol{\theta}$ is continuous and $w$ is nonconforming. The analysis of the other cases could be performed along similar lines.

It would be interesting to compare these new elements with the more classical $\mathrm{MITC}_{k}$ families (see [12] or [15]) and the elements in [5], as well as with the more recent methods such as [6-8,21,22,25-27].

Even more interesting would be the extension of these DG techniques to the treatment of shell problems. See for instance [24,9,17-20,22], and the references therein for a discussion of the difficulties in designing accurate and robust shell elements. We point out here that our elements, at least in the totally discontinuous version, use the same degrees of freedom for the rotations and the transverse displacement, which is usually considered as a very favorable feature for the discretization of shell problems in the Naghdi model.

The paper is organized as follows. After a section on notations and preliminaries, in Sec. 3 we recall the Reissner-Mindlin model and derive our family of methods in the fully discontinuous case. The corresponding error estimates are proved in Sec. 4. Finally, in Sec. 5, we present the case of continuous $\boldsymbol{\theta}$ and nonconforming $w$, together with its analysis.

\section{NOTATIONS AND PRELIMINARIES}

Let $\Omega \subset \mathbb{R}^{2}$ denote the domain occupied by the middle surface of the plate. We shall use the usual Sobolev spaces such as $H^{s}(T)$, with the 
corresponding semi-norm and norm denoted by $|\cdot|_{s, T}$ and $\|\cdot\|_{s, T}$, respectively. When $T=\Omega$ we just write $|\cdot|_{s}$ and $\|\cdot\|_{s}$. By convention, we use boldface type for the vector-valued analogues: $\boldsymbol{H}^{s}(\Omega)=\left[H^{s}(\Omega)\right]^{2}$. Occasionally we shall use calligraphic type for symmetric-tensor-valued analogues: $\mathcal{H}^{s}(\Omega)=\left[H^{s}(\Omega)\right]_{\text {sym }}^{2}$. We use parentheses $(\cdot, \cdot)$ to denote the inner product in any of the spaces $L^{2}(\Omega), \boldsymbol{L}^{2}(\Omega)$, or $\mathcal{L}^{2}(\Omega)$.

We shall assume that the domain $\Omega$ is a polygon and denote by $\mathcal{T}_{h}$ a decomposition of $\Omega$ into triangles $T$, by $\mathcal{E}_{h}$ the set of all the edges in $\mathcal{T}_{h}$, and by $\mathcal{E}_{h}^{0}$ the set of interior edges. We use the notation for piecewise polynomial spaces from [14], so

$$
\mathcal{L}_{k}^{s}\left(\mathcal{T}_{h}\right)=\left\{v \in H^{s}(\Omega):\left.v\right|_{T} \in \mathcal{P}_{k}(T), T \in \mathcal{T}_{h}\right\}
$$

with $\mathcal{P}_{k}(T)$ the set of polynomials of degree at most $k$ on $T$ (note that in this usage, calligraphic type does not refer to tensor-valued quantities).

Our finite elements will be discontinuous and so not contained in the space $H^{1}(\Omega)$, but rather in a piecewise Sobolev space

$$
H^{1}\left(\mathcal{T}_{h}\right):=\left\{v \in L^{2}(\Omega):\left.v\right|_{T} \in H^{1}(T), T \in \mathcal{T}_{h}\right\} .
$$

Differential operators can be applied to this space piecewise. We indicate this by a subscript $h$ on the operator. Thus, for example, the piecewise gradient operator $\nabla_{h}$ maps $H^{1}\left(\mathcal{T}_{h}\right)$ into $\boldsymbol{L}^{2}(\Omega)$ and the piecewise symmetric gradient, or infinitesimal strain, operator $\varepsilon_{h}$ maps $\boldsymbol{H}^{1}\left(\mathcal{T}_{h}\right)$ into $\mathcal{L}^{2}(\Omega)$. The space $H^{1}\left(\mathcal{T}_{h}\right)$ is equipped with the semi-norm $|v|_{1, h}=\left\|\nabla_{h} v\right\|_{0}$ and the corresponding norm $\|v\|_{1, h}^{2}=|v|_{1, h}^{2}+\|v\|_{0}^{2}$.

As is usual in the DG approach, we define the jump and average of a function in $H^{1}\left(\mathcal{T}_{h}\right)$ as a function on the union of the edges of the triangulation. Let $e$ be an internal edge of $\mathcal{T}_{h}$, shared by two elements $T^{+}$and $T^{-}$, and let $\boldsymbol{n}^{+}$and $\boldsymbol{n}^{-}$denote the unit normals to $e$, pointing outward from $T^{+}$and $T^{-}$, respectively. If $\varphi$ belongs to $H^{1}\left(\mathcal{T}_{h}\right.$ ) (or possibly the vector- or tensor-valued analogue), we define the average $\{\varphi\}$ on $e$ as usual:

$$
\{\varphi\}=\frac{\varphi^{+}+\varphi^{-}}{2} .
$$

For a scalar function $\varphi \in H^{1}\left(\mathcal{T}_{h}\right)$ we define its jump on $e$ as

$$
[\varphi]=\varphi^{+} \boldsymbol{n}^{+}+\varphi^{-} \boldsymbol{n}^{-},
$$

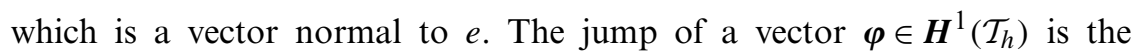
symmetric matrix-valued function given on $e$ by:

$$
[\varphi]=\varphi^{+} \odot n^{+}+\varphi^{-} \odot n^{-},
$$


where $\boldsymbol{\varphi} \odot \boldsymbol{n}=\left(\boldsymbol{\varphi} \boldsymbol{n}^{T}+\boldsymbol{n} \varphi^{T}\right) / 2$ is the symmetric part of the tensor product of $\boldsymbol{\varphi}$ and $\boldsymbol{n}$.

On a boundary edge, the average $\{\varphi\}$ is defined simply as the trace of $\varphi$, while for a scalar-valued function we define $[\varphi]$ to be $\varphi \boldsymbol{n}$ (with $\boldsymbol{n}$ the outward unit normal), and for a vector-valued function we define $[\varphi]=\varphi \odot$ $n$.

It is easy to check that (see, e.g. [3])

$$
\sum_{T \in \mathcal{T}_{h}} \int_{\partial T} \boldsymbol{\varphi} \cdot \boldsymbol{n}_{T} v d s=\sum_{e \in \mathcal{E}_{h}} \int_{e}\{\boldsymbol{\varphi}\} \cdot[v] d s, \quad \boldsymbol{\varphi} \in \boldsymbol{H}^{1}(\Omega), \quad v \in H^{1}\left(\mathcal{T}_{h}\right) .
$$

Similarly,

$$
\sum_{T \in \mathcal{T}_{h}} \int_{\partial T} \mathcal{S} \boldsymbol{n}_{T} \cdot \boldsymbol{\eta} d s=\sum_{e \in \mathcal{E}_{h}} \int_{e}\{\mathcal{S}\}:[\boldsymbol{\eta}] d s, \quad \mathcal{S} \in \mathcal{H}^{1}(\Omega), \quad \boldsymbol{\eta} \in \boldsymbol{H}^{1}\left(\mathcal{T}_{h}\right) .
$$

It is not difficult to see that both the above relations hold in more general situations. For instance, (2) also holds for $\boldsymbol{\varphi} \in \boldsymbol{H}(\operatorname{div} ; \Omega)$, where $\boldsymbol{H}(\operatorname{div} ; \Omega)$ is the space of vectors $\varphi \in \boldsymbol{L}^{2}(\Omega)$ with $\operatorname{div} \boldsymbol{\varphi} \in L^{2}(\Omega)$.

In the sequel we shall often use the following result (see [1,2]): let $T$ be a triangle, and let $e$ be an edge of $T$. Then there exists a positive constant $C$ only depending on the minimum angle of $T$ such that

$$
\|\varphi\|_{0, e}^{2} \leqslant C\left(|e|^{-1}\|\varphi\|_{0, T}^{2}+|e \| \varphi|_{1, T}^{2}\right), \quad \varphi \in H^{1}(T) .
$$

Clearly, (3) also holds for vector valued functions $\boldsymbol{\varphi} \in \boldsymbol{H}^{1}\left(\mathcal{T}_{h}\right)$.

\section{THE PROBLEM AND A DG DISCRETIZATION}

In this section we recall the Reissner-Mindlin plate model and derive a DG discretization of it.

Given the load $g$ in $L^{2}(\Omega)$ and the tensor of bending moduli $C$, the Reissner-Mindlin equations with clamped boundary determine the rotation $\boldsymbol{\theta}$, transverse displacement $w$, and scaled shear stress $\gamma$ by the equations

$$
\begin{aligned}
& -\operatorname{div} C \varepsilon(\boldsymbol{\theta})-\boldsymbol{\gamma}=0 \quad \text { in } \Omega, \\
& -\operatorname{div} \gamma=g \quad \text { in } \Omega, \\
& \nabla w-\boldsymbol{\theta}-\lambda^{-1} t^{2} \gamma=0 \quad \text { in } \Omega, \\
& \boldsymbol{\theta}=0, \quad w=0 \quad \text { on } \partial \Omega \text {. }
\end{aligned}
$$


Here $\varepsilon$ denotes the usual symmetric gradient operator, $\lambda$ the shear correction factor, and $t$ the plate thickness. Henceforth we will incorporate $\lambda$ in the thickness (still denoting it by $t$ ).

To obtain a weak mixed formulation of the system (4)-(7) we multiply (4) by a test function $\boldsymbol{\eta} \in \boldsymbol{H}_{0}^{1}(\Omega)$ and (5) by a test function $v \in H_{0}^{1}(\Omega)$, integrate by parts, and add the equations. Next, we multiply (6) by a test function $\boldsymbol{\tau} \in \boldsymbol{L}^{2}(\Omega)$ and integrate. We thus find that $(\boldsymbol{\theta}, w) \in \boldsymbol{H}_{0}^{1}(\Omega) \times H_{0}^{1}(\Omega)$ and $\boldsymbol{\gamma} \in \boldsymbol{L}^{2}(\Omega)$ satisfy

$$
\begin{aligned}
(\mathbf{C} \varepsilon(\boldsymbol{\theta}), \varepsilon(\boldsymbol{\eta}))+(\boldsymbol{\gamma}, \boldsymbol{\nabla} v-\boldsymbol{\eta}) & =(g, v), & & (\boldsymbol{\eta}, v) \in \boldsymbol{H}_{0}^{1}(\Omega) \times H_{0}^{1}(\Omega), \\
(\boldsymbol{\nabla} w-\boldsymbol{\theta}, \boldsymbol{\tau})-t^{2}(\boldsymbol{\gamma}, \boldsymbol{\tau}) & =0, & & \boldsymbol{\tau} \in \boldsymbol{L}^{2}(\Omega) .
\end{aligned}
$$

A natural way to discretize the Reissner-Mindlin system is to restrict the trial and test functions in this weak formulation to finite dimensional subspaces. That is, we choose finite dimensions subspaces $\boldsymbol{\Theta}_{h} \subset \boldsymbol{H}_{0}^{1}(\Omega)$, $W_{h} \subset H_{0}^{1}(\Omega)$, and $\boldsymbol{\Gamma}_{h} \subset \boldsymbol{L}^{2}(\Omega)$ and define $\left(\boldsymbol{\theta}_{h}, w_{h}\right) \in \boldsymbol{\Theta}_{h} \times W_{h}$ and $\gamma_{h} \in \boldsymbol{\Gamma}_{h}$ by the equations

$$
\begin{aligned}
\left(\mathbf{C} \varepsilon\left(\boldsymbol{\theta}_{h}\right), \varepsilon(\boldsymbol{\eta})\right)+\left(\gamma_{h}, \nabla v-\boldsymbol{\eta}\right) & =(g, v), & & (\boldsymbol{\eta}, v) \in \boldsymbol{\Theta}_{h} \times W_{h}, \\
\left(\boldsymbol{\nabla} w_{h}-\boldsymbol{\theta}_{h}, \boldsymbol{\tau}\right)-t^{2}\left(\gamma_{h}, \boldsymbol{\tau}\right) & =0, & & \boldsymbol{\tau} \in \boldsymbol{\Gamma}_{h} .
\end{aligned}
$$

In order to overcome the well-known problem of locking - the loss of accuracy for small plate thickness - this formulation is often generalized by the inclusion of a projection operator $\boldsymbol{P}_{h}: \boldsymbol{H}^{1}\left(\mathcal{T}_{h}\right) \rightarrow \boldsymbol{\Gamma}_{h}$ to obtain the system

$$
\begin{aligned}
\left(\mathbf{C}_{\varepsilon}\left(\boldsymbol{\theta}_{h}\right), \varepsilon(\boldsymbol{\eta})\right)+\left(\gamma_{h}, \boldsymbol{P}_{h}(\boldsymbol{\nabla} v-\boldsymbol{\eta})\right) & =(g, v), & & (\boldsymbol{\eta}, v) \in \boldsymbol{\Theta}_{h} \times W_{h}, \\
\left(\boldsymbol{P}_{h}\left(\boldsymbol{\nabla} w_{h}-\boldsymbol{\theta}_{h}\right), \boldsymbol{\tau}\right)-t^{2}\left(\gamma_{h}, \boldsymbol{\tau}\right) & =0, & & \boldsymbol{\tau} \in \boldsymbol{\Gamma}_{h} .
\end{aligned}
$$

(The method without $\boldsymbol{P}_{h}$ can be viewed as the special case where $\boldsymbol{P}_{h}$ is taken to be the $L^{2}$-projection onto $\boldsymbol{\Gamma}_{h}$.) A number of the most successful finite element methods for the Reissner-Mindlin system can be written in this form with appropriate choices for the spaces $\boldsymbol{\Theta}_{h}, W_{h}$, and $\boldsymbol{\Gamma}_{h}$ and the projection operator $\boldsymbol{P}_{h}$. However, simple choices of the finite element spaces have been found to be unsuccessful even with the use of a projection operator. For example, the choice of continuous piecewise linear functions for $\boldsymbol{\Theta}_{h}$ and $W_{h}$ and piecewise constant functions for $\boldsymbol{\Gamma}_{h}$ seems natural, but does not give a good method. In this paper we will show that very simple discontinuous finite element spaces can be used.

To derive a finite element method for the Reissner-Mindlin system based on discontinuous elements, we proceed as before testing (4) against a test function $\boldsymbol{\eta}$ and (5) against a test function $v$, integrating by parts, 
and adding, with the difference that now $\boldsymbol{\eta}$ and $v$ may be discontinuous across element boundaries, that is, they belong to $\boldsymbol{H}^{1}\left(\mathcal{T}_{h}\right)$ and $H^{1}\left(\mathcal{T}_{h}\right)$, respectively. Thus we obtain

$$
\begin{aligned}
& \left(\mathbf{C} \varepsilon_{h}(\boldsymbol{\theta}), \varepsilon_{h}(\boldsymbol{\eta})\right)-\sum_{e \in \mathcal{E}_{h}} \int_{e}\left\{\mathbf{C} \varepsilon_{h}(\boldsymbol{\theta})\right\}:[\boldsymbol{\eta}] d s+\left(\boldsymbol{\gamma}, \nabla_{h} v-\boldsymbol{\eta}\right) \\
& -\sum_{e \in \mathcal{E}_{h}} \int_{e}\{\boldsymbol{\gamma}\} \cdot[v] d s=(g, v), \quad(\boldsymbol{\eta}, v) \in \boldsymbol{H}^{1}\left(\mathcal{T}_{h}\right) \times H^{1}\left(\mathcal{T}_{h}\right), \\
& \quad\left(\nabla_{h} w-\boldsymbol{\theta}, \boldsymbol{\tau}\right)-t^{2}(\boldsymbol{\gamma}, \boldsymbol{\tau})=0, \quad \boldsymbol{\tau} \in L^{2}(\Omega) .
\end{aligned}
$$

The two terms in the first equation involving integrals over the edges, which did not appear in (8), arise from the integration by parts and are necessary to maintain consistency. We now proceed as is common for DG methods. First, we add terms to symmetrize this formulation so that it is adjoint-consistent as well. Second, to stabilize the method, we add interior penalty terms $p_{\Theta}(\boldsymbol{\theta}, \boldsymbol{\eta})$ and $p_{W}(w, v)$ in which the functions $p_{\Theta}$ and $p_{W}$ will depend only on the jumps of their arguments. Since $[\boldsymbol{\theta}]=0$ and $[w]=0$, we find that $\theta, w$, and $\gamma$ satisfy

$$
\begin{aligned}
& \left(\mathbf{C} \varepsilon_{h}(\boldsymbol{\theta}), \varepsilon_{h}(\boldsymbol{\eta})\right)-\sum_{e \in \mathcal{E}_{h}} \int_{e}\left(\left\{\mathbf{C} \varepsilon_{h}(\boldsymbol{\theta})\right\}:[\boldsymbol{\eta}] d s+[\boldsymbol{\theta}]:\left\{C \varepsilon_{h}(\boldsymbol{\eta})\right\}\right) d s \\
& +\left(\boldsymbol{\gamma}, \boldsymbol{\nabla}_{h} v-\boldsymbol{\eta}\right)-\sum_{e \in \mathcal{E}_{h}} \int_{e}\{\boldsymbol{\gamma}\} \cdot[v] d s+p_{\Theta}(\boldsymbol{\theta}, \boldsymbol{\eta})+p_{W}(w, v) \\
& =(g, v), \quad(\boldsymbol{\eta}, v) \in \boldsymbol{H}^{2}\left(\mathcal{T}_{h}\right) \times H^{2}\left(\mathcal{T}_{h}\right), \\
& \left(\nabla_{h} w-\boldsymbol{\theta}, \boldsymbol{\tau}\right)-\sum_{e \in \mathcal{E}_{h}} \int_{e}[w] \cdot\{\boldsymbol{\tau}\} d s-t^{2}(\boldsymbol{\gamma}, \boldsymbol{\tau})=0, \quad \boldsymbol{\tau} \in \boldsymbol{H}^{1}\left(\mathcal{T}_{h}\right) .
\end{aligned}
$$

To obtain a DG discretization, we choose finite dimensional subspaces $\boldsymbol{\Theta}_{h} \subset \boldsymbol{H}^{2}\left(\mathcal{T}_{h}\right), W_{h} \subset H^{2}\left(\mathcal{T}_{h}\right)$, and $\boldsymbol{\Gamma}_{h} \subset \boldsymbol{H}^{1}\left(\mathcal{T}_{h}\right)$ and, in analogy with the continuous Galerkin case, we incorporate a projection operator $\boldsymbol{P}_{h}: \boldsymbol{H}^{1}\left(\mathcal{T}_{h}\right) \rightarrow$ $\boldsymbol{\Gamma}_{h}$, so that the method takes the form: Find $\left(\boldsymbol{\theta}_{h}, w_{h}\right) \in \boldsymbol{\Theta}_{h} \times W_{h}$ and $\gamma_{h} \in$ $\Gamma_{h}$ such that

$$
\begin{aligned}
& \left(\mathbf{C} \varepsilon_{h}\left(\boldsymbol{\theta}_{h}\right), \varepsilon_{h}(\boldsymbol{\eta})\right)-\sum_{e \in \mathcal{E}_{h}} \int_{e}\left(\left\{\mathbf{C} \varepsilon_{h}\left(\boldsymbol{\theta}_{h}\right)\right\}:[\boldsymbol{\eta}] d s+\left[\boldsymbol{\theta}_{h}\right]:\left\{\mathbf{C} \varepsilon_{h}(\boldsymbol{\eta})\right\}\right) d s \\
& +\left(\gamma_{h}, \boldsymbol{P}_{h}\left(\nabla_{h} v-\boldsymbol{\eta}\right)\right)-\sum_{e \in \mathcal{E}_{h}} \int_{e}\left\{\gamma_{h}\right\} \cdot[v] d s+p_{\Theta}\left(\boldsymbol{\theta}_{h}, \boldsymbol{\eta}\right)+p_{W}\left(w_{h}, v\right) \\
& =(g, v), \quad(\boldsymbol{\eta}, v) \in \boldsymbol{\Theta}_{h} \times W_{h},
\end{aligned}
$$




$$
\left(\boldsymbol{P}_{h}\left(\nabla_{h} w_{h}-\boldsymbol{\theta}_{h}\right), \boldsymbol{\tau}\right)-\sum_{e \in \mathcal{E}_{h}} \int_{e}\left[w_{h}\right] \cdot\{\boldsymbol{\tau}\} d s-t^{2}\left(\gamma_{h}, \boldsymbol{\tau}\right)=0, \quad \boldsymbol{\tau} \in \boldsymbol{\Gamma}_{h} .
$$

For any choice of the finite element spaces $\boldsymbol{\Theta}_{h}, W_{h}$, and $\boldsymbol{\Gamma}_{h}$, and any interior penalty functions $p_{\Theta}$ and $p_{W}$ depending only on the jumps of their arguments, this gives a consistent finite element method when the projection operator $\boldsymbol{P}_{h}$ is simply the $L^{2}$-projection onto $\boldsymbol{\Gamma}_{h}$. Most other choices of $\boldsymbol{P}_{h}$ introduce a consistency error just as for continuous Galerkin methods.

The numerical method we will consider is of the form (12), (13). To complete the specification of the method we need to specify three things: the finite element spaces $\boldsymbol{\Theta}_{h}, W_{h}$, and $\boldsymbol{\Gamma}_{h}$; the interior penalty forms $p_{\Theta}$ and $p_{W}$; and the projection operator $\boldsymbol{P}_{h}$. For the finite element spaces we make a simple choice, namely for an integer $k \geqslant 1$ we use fully discontinuous piecewise polynomials of degree $k$ to discretize $\boldsymbol{\theta}$ and $\boldsymbol{w}$, and of degree $k-1$ for $\gamma$. Using the notation introduced in (1),

$$
\boldsymbol{\Theta}_{h}={ }_{k}^{\vee 0}\left(\mathcal{T}_{h}\right), \quad W_{h}=\mathcal{L}_{k}^{0}\left(\mathcal{T}_{h}\right), \quad \boldsymbol{\Gamma}_{h}={ }_{k-1}^{\sim 0}\left(\mathcal{T}_{h}\right) .
$$

Note that this choice ensures that

$$
\nabla_{h}\left(W_{h}\right) \subset \Gamma_{h}
$$

an important relation for this method as for many discretizations of the Reissner-Mindlin system. This, of course, implies that, for any projection operator $\boldsymbol{P}_{h}: \boldsymbol{H}^{1}\left(\mathcal{T}_{h}\right) \rightarrow \boldsymbol{\Gamma}_{h}, \boldsymbol{P}_{h} \nabla_{h} v=\nabla_{h} v$ for all $v \in W_{h}$.

We make a standard choice for the interior penalty term $p_{\Theta}$ :

$$
p_{\Theta}(\boldsymbol{\theta}, \boldsymbol{\eta})=\sum_{e \in \mathcal{E}_{h}} \frac{\kappa^{\Theta}}{|e|} \int_{e}[\boldsymbol{\theta}]:[\boldsymbol{\eta}] d s
$$

so that $p_{\Theta}(\boldsymbol{\eta}, \boldsymbol{\eta})$ can be viewed as a measure of the deviation of $\boldsymbol{\eta}$ from being continuous. The parameter $\kappa^{\Theta}$ is a positive constant to be chosen; it must be sufficiently large to ensure stability. For $p_{W}$ we use a weaker penalization:

$$
p_{W}(w, v)=\sum_{e \in \mathcal{E}_{h}} \frac{\kappa^{W}}{|e|} \int_{e} \boldsymbol{Q}_{e}[w] \cdot \boldsymbol{Q}_{e}[v] d s
$$

where $\boldsymbol{Q}_{e}$ is the $L^{2}$-projection onto polynomials of degree $k-1$ on the edge and $\kappa^{W}$ is again a positive constant to be chosen. Thus we penalize 
the deviation of $w$ from the usual nonconforming degree $k$ finite element space rather than the deviation from continuity.

Finally, we need to specify the projection operator $\boldsymbol{P}_{h}$. In the lowest order case, $k=1$, we simply choose the $L^{2}$-projection onto the piecewise constant space $\mathcal{L}_{0}^{0}\left(\mathcal{T}_{h}\right)$. For $k>1$ the definition of $\boldsymbol{P}_{h}$ is more complicated and requires some notation and a lemma. For any odd integer $k>1$ and any triangle $T$, define

$$
\Gamma^{*}(T)=\left\{\boldsymbol{\tau}+\operatorname{curl}\left(b_{T} v\right) \mid \boldsymbol{\tau} \in \mathcal{P}_{k-1}(T), \operatorname{div} \boldsymbol{\tau} \in \mathcal{P}_{k-3}(T), v \in \mathcal{P}_{k-2}(T)\right\} .
$$

Here $b_{T}$ is the cubic bubble given by $\lambda_{1} \lambda_{2} \lambda_{3}$ where the $\lambda_{i}$ are the barycentric coordinate functions on $T$, and $\operatorname{curl} v:=(-\partial v / \partial y, \partial v / \partial x)$ (with formal adjoint $\left.\operatorname{rot} \delta:=\partial \delta_{1} / \partial y-\partial \delta_{2} / \partial x\right)$. For $k=1$ we interpret $\Gamma^{*}(T)=\mathcal{P}_{0}(T)$. Note that $\operatorname{dim} \Gamma^{*}(T)=\operatorname{dim} \mathcal{P}_{k-1}(T)$.

Lemma 3.1. Let $k$ be a positive odd integer and $T$ a triangle. If $\delta \in$ $\mathcal{P}_{k-1}(T)$ satisfies $\int_{T} \boldsymbol{\delta} \cdot \boldsymbol{\rho} d x=0$ for all $\boldsymbol{\rho} \in \boldsymbol{\Gamma}^{*}(T)$, then $\boldsymbol{\delta}=0$.

Proof. This is obvious for $k=1$ so we assume $k \geqslant 3$. By integration by parts, we have $\int_{T}(\operatorname{rot} \delta) b_{T} v d x=0$ for all $v \in \mathcal{P}_{k-2}(T)$. In particular, we can take $v=\operatorname{rot} \delta$ and conclude that $\operatorname{rot} \delta=0$. Therefore $\delta=\nabla \psi$ for some $\psi \in \mathcal{P}_{k}(T)$ which we can normalize to have mean value 0 on $\partial T$. Now, given an arbitrary $q \in \mathcal{P}_{k-2}(T)$ and an arbitrary piecewise polynomial $\mu$ of degree $k-1$ on $\partial T$ (that is, $\mu$ restricts to a polynomial of degree $k-1$ on each edge of $T$ ), we have that the equation

$$
\operatorname{div} \boldsymbol{\tau}=q \quad \text { in } T, \quad \boldsymbol{\tau} \cdot \boldsymbol{n}=\mu \quad \text { on } \partial T
$$

has a solution $\tau \in \mathcal{P}_{k-1}(T)$ if and only if $\int_{T} q d x=\int_{\partial T} \mu d s$ (This can be checked by counting dimensions and noting that $\tau$ satisfies (16) for $q=0$, $\mu=0$ if and only if $\boldsymbol{\tau}=\operatorname{curl}\left(b_{T} p\right)$ for some $\left.p \in \mathcal{P}_{k-3}(T)\right)$. Taking $q=0$ and $\mu$ an arbitrary piecewise polynomial of mean value 0 on $\partial T$, we can solve (16) to find $\boldsymbol{\tau} \in \Gamma^{*}(T)$. Then integration by parts gives

$$
0=\int_{T} \nabla \psi \cdot \tau d x=\int_{\partial T} \psi \mu d s .
$$

This, together with our normalization $\int_{\partial T} \psi d s=0$ shows that $\left.\psi\right|_{\partial T}$ is orthogonal to all piecewise polynomials of degree $k-1$. Therefore on each edge $\psi$ must be a multiple of the Legendre polynomial of degree $k$ and hence it mush change sign exactly $k$ times on each edge (unless it is identically 0 ). The global continuity of $\psi$, however, rules out an odd number $(3 k)$ of sign changes, so we conclude that $\psi=0$ on $\partial T$, i.e., $\psi=$ $b_{T} \phi$ for some $\phi \in \mathcal{P}_{k-3}(T)$. Now take $q=\phi, \mu=$ constant on $\partial T$ in (16). 
The resulting $\boldsymbol{\tau}$ belongs to $\Gamma^{*}(T)$ and so is orthogonal to $\delta=\nabla\left(b_{T} \phi\right)$, and now integration by parts immediately implies that $\phi=0$.

Let

$$
\boldsymbol{\Gamma}_{h}^{*}=\left\{\boldsymbol{\tau} \in \boldsymbol{L}^{2}(\Omega)|\boldsymbol{\tau}|_{T} \in \boldsymbol{\Gamma}^{*}(T), T \in \mathcal{T}_{h}\right\} .
$$

In view of the lemma, we may define $\boldsymbol{P}_{h}: \boldsymbol{L}^{2}(\Omega) \rightarrow \boldsymbol{\Gamma}_{h}$ by

$$
\left(\boldsymbol{\delta}-\boldsymbol{P}_{h} \boldsymbol{\delta}, \boldsymbol{\tau}\right)=0, \quad \boldsymbol{\tau} \in \boldsymbol{\Gamma}_{h}^{*} .
$$

\section{ERROR ANALYSIS}

Having completed the specification of our family of DG methods (one for each positive odd integer $k$ ), in this section we state and prove the basic error estimates for the methods. For this purpose we first define norms

$$
\begin{aligned}
\|\boldsymbol{\eta}\|_{\Theta}^{2}:=\|\boldsymbol{\eta}\|_{1, h}^{2}+\sum_{e \in \mathcal{E}_{h}}\left(\frac{1}{|e|}\|[\boldsymbol{\eta}]\|_{0, e}^{2}+|e|\left\|\left\{\mathbf{C}_{\varepsilon_{h}}(\boldsymbol{\eta})\right\}\right\|_{0, e}^{2}\right), \\
\|v\|_{W}^{2}:=|v|_{1, h}^{2}+\sum_{e \in \mathcal{E}_{h}} \frac{1}{|e|}\|[v]\|_{0, e}^{2} \\
\|\boldsymbol{\tau}\|_{\Gamma}^{2}:=\|\boldsymbol{\tau}\|_{0}^{2}+\sum_{e \in \mathcal{E}_{h}}|e|\|\{\boldsymbol{\tau}\}\|_{0, e}^{2}
\end{aligned}
$$

for $\boldsymbol{\eta} \in \boldsymbol{H}^{2}\left(\mathcal{T}_{h}\right), v \in H^{1}\left(\mathcal{T}_{h}\right)$, and $\boldsymbol{\tau} \in \boldsymbol{H}^{1}\left(\mathcal{T}_{h}\right)$.

Remark 4.1. If we replace $[v]$ in (20) with its projection into some polynomial space on the edge, we obtain an equivalent norm. That is,

$$
\begin{aligned}
\|v\|_{W}^{2} & \approx|v|_{1, h}^{2}+\sum_{e \in \mathcal{E}_{h}} \frac{1}{|e|}\left\|\boldsymbol{Q}_{e}([v])\right\|_{0, e}^{2} \\
& \approx|v|_{1, h}^{2}+\sum_{e \in \mathcal{E}_{h}} \frac{1}{|e|}\left\|\boldsymbol{Q}_{e}^{0}([v])\right\|_{0, e}^{2}, \quad v \in H^{1}\left(\mathcal{T}_{h}\right),
\end{aligned}
$$

where $\boldsymbol{Q}_{e}$ is, as above, the $L^{2}$-projection onto polynomials of degree $k-1$ on the edge $e$, and $\boldsymbol{Q}_{e}^{0}$ the $L^{2}$-projection onto constants on $e$, and the constants of equivalence depend only on the minimum angle of the triangulation. Obviously

$$
|v|_{1, h}^{2}+\sum_{e \in \mathcal{E}_{h}} \frac{1}{|e|}\left\|\boldsymbol{Q}_{e}^{0}([v])\right\|_{0, e}^{2} \leqslant|v|_{1, h}^{2}+\sum_{e \in \mathcal{E}_{h}} \frac{1}{|e|}\left\|\boldsymbol{Q}_{e}([v])\right\|_{0, e}^{2} \leqslant\|v\|_{W}^{2},
$$


so, to establish (22), we need only show that

$$
\sum_{e \in \mathcal{E}_{h}} \frac{1}{|e|}\|[v]\|_{0, e}^{2} \leqslant C|v|_{1, h}^{2}+\sum_{e \in \mathcal{E}_{h}} \frac{1}{|e|}\left\|\boldsymbol{Q}_{e}^{0}([v])\right\|_{0, e}^{2} .
$$

Now if $v \in H^{1}(T)$ for some triangle $T$ with edge $e$ and $Q_{e}^{0} v$ denotes the average of $v$ on $e$ (i.e., the $L^{2}$-projection into constants of its trace on $e$ ), we have

$$
\frac{1}{|e|}\left\|v-Q_{e}^{0} v\right\|_{0, e}^{2} \leqslant C\left(\frac{1}{|e|^{2}}\left\|v-Q_{e}^{0} v\right\|_{0, T}^{2}+\|\nabla v\|_{0, T}^{2}\right) \leqslant C\|\nabla v\|_{0, T}^{2},
$$

where we have used (3) and a simple approximation result. It follows that, for $v \in H^{1}\left(\mathcal{T}_{h}\right)$

$$
\sum_{e \in \mathcal{E}_{h}} \frac{1}{|e|}\left\|[v]-Q_{e}^{0}([v])\right\|_{0, e}^{2} \leqslant C|v|_{1, h}^{2}
$$

and so

$$
\begin{aligned}
\sum_{e \in \mathcal{E}_{h}} \frac{1}{|e|}\|[v]\|_{0, e}^{2} & =\sum_{e \in \mathcal{E}_{h}} \frac{1}{|e|}\left(\left\|[v]-\boldsymbol{Q}_{e}^{0}([v])\right\|_{0, e}^{2}+\left\|\boldsymbol{Q}_{e}^{0}([v])\right\|_{0, e}^{2}\right) \\
& \leqslant C|v|_{1, h}^{2}+\sum_{e \in \mathcal{E}_{h}} \frac{1}{|e|}\left\|\boldsymbol{Q}_{e}^{0}([v])\right\|_{0, e}^{2}
\end{aligned}
$$

as desired.

The following theorem is the principal result of the paper.

Theorem 4.2. Let $\boldsymbol{\theta}, w, \gamma$ solve the Reissner-Mindlin system (8), (9). Let $k$ be a positive odd integer and suppose that the penalty parameter $\kappa^{\Theta}$ is sufficiently large and the penalty parameter $\kappa^{W}$ is positive. Then there exists a unique solution $\boldsymbol{\theta}_{h}, w_{h}, \gamma_{h}$ to the DG method (12) and (13). Moreover, there exists a constant $C$, independent of $h$ and $t$, such that

$$
\begin{gathered}
\left\|\boldsymbol{\theta}-\boldsymbol{\theta}_{h}\right\|_{\Theta}+\left\|w-w_{h}\right\|_{W}+\varpi\left\|\gamma-\gamma_{h}\right\|_{\Gamma} \\
\leqslant C h^{k}\left(\|\boldsymbol{\theta}\|_{k+1}+\|w\|_{k+1}+\|\gamma\|_{k}\right) .
\end{gathered}
$$

Remark 4.3. This estimate is clearly optimal with respect to the power of $h$ and with respect to the regularity of $\boldsymbol{\theta}$ and $w$. With respect to the regularity of $\gamma$ one might hope to replace $\|\gamma\|_{k}$ with $t\|\gamma\|_{k}+\|\gamma\|_{k-1}+$ $\|\operatorname{div} \gamma\|_{k-1}$ on the right-hand side. However, such an estimate does not follow from the current analysis. We will however be able to prove it, in the last section, for the continuous-nonconforming case. 
We now turn to the proof Theorem 4.2, beginning by introducing some notation. Let

$$
\begin{aligned}
a_{h}(\boldsymbol{\theta}, \boldsymbol{\eta})= & \left(\mathbf{C} \varepsilon_{h}(\boldsymbol{\theta}), \varepsilon_{h}(\boldsymbol{\eta})\right) \\
& -\sum_{e \in \mathcal{E}_{h}} \int_{e}\left(\left\{\mathbf{C} \varepsilon_{h}(\boldsymbol{\theta})\right\}:[\boldsymbol{\eta}]+[\boldsymbol{\theta}]:\left\{\mathbf{C} \varepsilon_{h}(\boldsymbol{\eta})\right\}\right) d s+p_{\Theta}(\boldsymbol{\theta}, \boldsymbol{\eta}), \\
j(\boldsymbol{\tau}, v)= & \sum_{e \in \mathcal{E}_{h}} \int_{e}\{\boldsymbol{\tau}\} \cdot[v] d s .
\end{aligned}
$$

Clearly we have

$$
\begin{aligned}
a_{h}(\boldsymbol{\theta}, \boldsymbol{\eta}) \leqslant C\|\boldsymbol{\theta}\|_{\Theta}\|\boldsymbol{\eta}\|_{\Theta}, & \quad \boldsymbol{\theta}, \boldsymbol{\eta} \in \boldsymbol{H}^{2}\left(\mathcal{T}_{h}\right), \\
j(\boldsymbol{\tau}, v) \leqslant C\|\boldsymbol{\tau}\|_{\Gamma}\|v\|_{W}, & v \in H^{1}\left(\mathcal{T}_{h}\right), \quad \boldsymbol{\tau} \in \boldsymbol{H}^{1}\left(\mathcal{T}_{h}\right) .
\end{aligned}
$$

In this notation we may rewrite (11) as

$$
\begin{aligned}
& a_{h}(\boldsymbol{\theta}, \boldsymbol{\eta})+\left(\gamma, \nabla_{h} v-\boldsymbol{\eta}\right)-j(\gamma, v)+p_{W}(w, v)=(g, v), \\
& \quad(\boldsymbol{\eta}, v) \in \boldsymbol{H}^{2}\left(\mathcal{T}_{h}\right) \times H^{2}\left(\mathcal{T}_{h}\right), \\
& \left(\nabla_{h} w-\boldsymbol{\theta}, \boldsymbol{\tau}\right)-j(\boldsymbol{\tau}, w)-t^{2}(\boldsymbol{\gamma}, \boldsymbol{\tau})=0, \quad \boldsymbol{\tau} \in \boldsymbol{H}^{1}\left(\mathcal{T}_{h}\right),
\end{aligned}
$$

and (12) and (13) as

$$
\begin{aligned}
& a_{h}\left(\boldsymbol{\theta}_{h}, \boldsymbol{\eta}\right)+\left(\gamma_{h}, \nabla_{h} v-\boldsymbol{P}_{h} \boldsymbol{\eta}\right)-j\left(\gamma_{h}, v\right)+p_{W}\left(w_{h}, v\right)=(g, v), \\
& \quad(\boldsymbol{\eta}, v) \in \boldsymbol{\Theta}_{h} \times W_{h}, \\
& \left(\nabla_{h} w_{h}-\boldsymbol{P}_{h} \boldsymbol{\theta}_{h}, \boldsymbol{\tau}\right)-j\left(\boldsymbol{\tau}, w_{h}\right)-t^{2}\left(\gamma_{h}, \boldsymbol{\tau}\right)=0, \quad \boldsymbol{\tau} \in \boldsymbol{\Gamma}_{h} .
\end{aligned}
$$

Defining a lifting operator $\boldsymbol{J}: \boldsymbol{H}^{1}\left(\mathcal{T}_{h}\right) \rightarrow \boldsymbol{\Gamma}_{h}$ by the equation

$$
(\boldsymbol{J}(v), \boldsymbol{\tau})=j(\boldsymbol{\tau}, v), \quad \boldsymbol{\tau} \in \boldsymbol{\Gamma}_{h},
$$

we can eliminate $\gamma_{h}$ in (29):

$$
\gamma_{h}=t^{-2}\left(\nabla_{h} w_{h}-\boldsymbol{J}\left(w_{h}\right)-\boldsymbol{P}_{h} \boldsymbol{\theta}_{h}\right) .
$$

Substituting in (28), we obtain an alternate formulation of the method:

$$
\begin{aligned}
a_{h}\left(\boldsymbol{\theta}_{h}, \boldsymbol{\eta}\right) & +t^{-2}\left(\nabla_{h} w_{h}-\boldsymbol{J}\left(w_{h}\right)-\boldsymbol{P}_{h} \boldsymbol{\theta}_{h}, \nabla_{h} v-\boldsymbol{J}(v)-\boldsymbol{P}_{h} \boldsymbol{\eta}\right) \\
& +p_{W}\left(w_{h}, v\right)=(g, v), \quad \boldsymbol{\eta} \in \boldsymbol{\Theta}_{h}, \quad v \in W_{h} .
\end{aligned}
$$


The following estimate for $\boldsymbol{J}$ will play an important role in the analysis. (Here and throughout the sequel we continue to denote by $C$ a generic constant which may depend on the mesh through its shape regularity but not otherwise and which is independent of $t$.)

\section{Proposition 4.4.}

$$
\|\boldsymbol{J}(v)\|_{\Gamma}^{2} \leqslant C \sum_{e \in \mathcal{E}_{h}} \frac{1}{|e|}\left\|\boldsymbol{Q}_{e}[v]\right\|_{0, e}^{2}, \quad v \in W_{h} .
$$

Proof. First we note that, by a local inverse inequality,

$$
\|\boldsymbol{\tau}\|_{\Gamma} \leqslant C\|\boldsymbol{\tau}\|_{0}, \quad \boldsymbol{\tau} \in \boldsymbol{\Gamma}_{h} .
$$

Now

$$
\begin{aligned}
\|\boldsymbol{J}(v)\|_{0}^{2} & =(\boldsymbol{J}(v), \boldsymbol{J}(v))=j(\boldsymbol{J}(v), v)=\sum_{e \in \mathcal{E}_{h}} \int_{e}\{\boldsymbol{J}(v)\} \cdot[v] d s \\
& =\sum_{e \in \mathcal{E}_{h}} \int_{e}\{\boldsymbol{J}(v)\} \cdot \boldsymbol{Q}_{e}[v] d s .
\end{aligned}
$$

Therefore

$$
\begin{aligned}
\|\boldsymbol{J}(v)\|_{0}^{2} & \leqslant\left(\sum_{e \in \mathcal{E}_{h}}|e|\|\{\boldsymbol{J}(v)\}\|_{0, e}^{2}\right)^{1 / 2}\left(\sum_{e \in \mathcal{E}_{h}} \frac{1}{|e|}\left\|\boldsymbol{Q}_{e}[v]\right\|_{0, e}^{2}\right)^{1 / 2} \\
& \leqslant\|\boldsymbol{J}(v)\|_{\Gamma}\left(\sum_{e \in \mathcal{E}_{h}} \frac{1}{|e|}\left\|\boldsymbol{Q}_{e}[v]\right\|_{0, e}^{2}\right)^{1 / 2}
\end{aligned}
$$

and so the proposition follows using (32).

Propositions 4.5 and 4.6 are analogues of Poincarés inequality and Korn's inequality for piecewise smooth functions.

Proposition 4.5.

$$
\|v\|_{0} \leqslant C\|v\|_{W}, \quad v \in H^{1}\left(\mathcal{T}_{h}\right) .
$$

Proof. The result is well known. See for instance [2] or the more general results of [10]. 


\section{Proposition 4.6.}

$$
\|\boldsymbol{\eta}\|_{1, h}^{2} \leqslant C\left(\sum_{T \in \mathcal{T}_{h}}\|\varepsilon(\boldsymbol{\eta})\|_{0, T}^{2}+\sum_{e \in \mathcal{E}_{h}} \frac{1}{|e|}\|[\boldsymbol{\eta}]\|_{0, e}^{2}\right), \quad \boldsymbol{\eta} \in \boldsymbol{H}^{1}\left(\mathcal{T}_{h}\right) .
$$

Proof. The result follows immediately using formula (1.12) of [11] to bound the $H^{1}\left(\mathcal{T}_{h}\right)$ seminorm of $\boldsymbol{\eta}$, and then using (1.3) of [10] to bound the $L^{2}$ norm. Note that, unlike in [10] and [11], our jump term includes integrals over edges in $\Gamma$, which allow us to avoid the integrals on $\Gamma$ appearing there.

Using Proposition 4.6, (3), and an inverse inequality, it is straightforward to verify the following proposition.

Proposition 4.7. There exist positive constants $\kappa_{0}$ and $\alpha$ depending only on the polynomial degree $k$ and the shape regularity of the partition $\mathcal{T}_{h}$, such that: if the constant $\kappa^{\Theta} \geqslant \kappa_{0}$ (where $\kappa^{\Theta}$ is the penalty parameter appearing in (15)), then

$$
a_{h}(\boldsymbol{\eta}, \boldsymbol{\eta}) \geqslant \alpha\|\boldsymbol{\eta}\|_{\Theta}^{2}, \quad \boldsymbol{\eta} \in \boldsymbol{\Theta}_{h} .
$$

To proceed with the analysis we define, for $\boldsymbol{\theta} \in \boldsymbol{H}^{1}(\Omega), w \in H^{1}(\Omega)$, and $\gamma \in \boldsymbol{L}^{2}(\Omega)$, approximations $\boldsymbol{\theta}_{I} \in \boldsymbol{\Theta}_{h}, w_{I} \in W_{h}$, and $\gamma_{I} \in \boldsymbol{\Gamma}_{h}$. For $\boldsymbol{\theta}_{I}$ we simply take the $L^{2}$-projection of $\boldsymbol{\theta}$ onto $\boldsymbol{\Theta}_{h}$. Since $\boldsymbol{\Gamma}_{h}^{*} \subset \boldsymbol{\Theta}_{h}$, an obvious (but important) consequence is that

$$
\boldsymbol{P}_{h} \boldsymbol{\theta}=\boldsymbol{P}_{h} \boldsymbol{\theta}_{I}, \quad \boldsymbol{\theta} \in H^{1}(\Omega) .
$$

Of course we have

$$
\left\|\boldsymbol{\theta}-\boldsymbol{\theta}_{I}\right\|_{\Theta} \leqslant C h^{k}\|\boldsymbol{\theta}\|_{k+1}
$$

For $w_{I}$ we use a standard nonconforming $\mathcal{P}_{k}$ interpolant. Namely on each triangle $T$ we define $\left.w_{I}\right|_{T} \in \mathcal{P}_{k}(T)$ by

$$
\begin{array}{ll}
\int_{e}\left(w-w_{I}\right) \mu d s=0, & \mu \in \mathcal{P}_{k-1}(e) \quad \text { for each edge } e \text { of } T, \\
\int_{T}\left(w-w_{I}\right) v d x=0, & v \in \mathcal{P}_{k-3}(T) .
\end{array}
$$

Note that

$$
\int_{T} \nabla\left(w-w_{I}\right) \cdot \boldsymbol{\tau} d x=-\int_{T}\left(w-w_{I}\right) \operatorname{div} \boldsymbol{\tau} d x+\int_{\partial T}\left(w-w_{I}\right) \boldsymbol{\tau} \cdot \boldsymbol{n} d s,
$$


which vanishes if $\boldsymbol{\tau} \in \mathcal{P}_{k-1}(T)$ with $\operatorname{div} \boldsymbol{\tau} \in \mathcal{P}_{k-3}(T)$ and certainly if $\boldsymbol{\tau}=$ $\operatorname{curl}\left(b_{T} v\right)$ for some $v$. Thus

$$
\boldsymbol{P}_{h}(\nabla w)=\boldsymbol{P}_{h}\left(\nabla_{h} w_{I}\right)=\nabla_{h} w_{I}, \quad w \in H^{1}(\Omega)
$$

with the last equality coming from (14). Standard approximation theory gives

$$
\left\|w-w_{I}\right\|_{W} \leqslant C h^{k}\|w\|_{k+1} .
$$

We also note that (38) implies that $\boldsymbol{Q}_{e}\left[w-w_{I}\right]=0$ on every edge $e$. Hence,

$$
p_{W}\left(w-w_{I}, v_{h}\right)=0, \quad v_{h} \in W_{h} .
$$

Finally we define $\gamma_{I}=\boldsymbol{P}_{h} \gamma$. Standard approximation arguments establish that

$$
\left\|\gamma-\gamma_{I}\right\|_{\Gamma} \leqslant C h^{k}\|\gamma\|_{k} .
$$

Most importantly, (36) and (40) together imply that if $\gamma=t^{-2}(\nabla w-\theta)$, then

$$
\gamma_{I}=t^{-2}\left(\nabla_{h} w_{I}-\boldsymbol{P}_{h} \boldsymbol{\theta}_{I}\right) .
$$

Following ideas from Duran and Liebermann [21], our analysis will rely on this last relation. Also important, but specific to the case of discontinuous elements, is the relation

$$
j\left(\boldsymbol{\tau}, w_{I}\right)=0, \quad w \in H^{1}(\Omega), \quad \boldsymbol{\tau} \in \boldsymbol{\Gamma}_{h},
$$

or, equivalently,

$$
\boldsymbol{J}\left(w_{I}\right)=0,
$$

which follows directly from (38).

We will bound the error between the exact solution $\boldsymbol{\theta}, w, \gamma$, determined by (26) and (27), and the Galerkin solution $\boldsymbol{\theta}_{h}, w_{h}, \gamma_{h}$, determined by (28) and (29), in terms of the errors in $\boldsymbol{\theta}_{I}, w_{I}$, and $\gamma_{I}$ which can in turn be bounded as in (37), (41), and (43). Let

$$
\boldsymbol{\theta}_{\delta}=\boldsymbol{\theta}_{h}-\boldsymbol{\theta}_{I}, \quad w_{\delta}=w_{h}-w_{I}, \quad \gamma_{\delta}=\gamma_{h}-\gamma_{I} .
$$

From (31), (44), and (45) we have

$$
\boldsymbol{P}_{h} \boldsymbol{\theta}_{\delta}=-t^{2} \gamma_{\delta}+\nabla_{h} w_{\delta}-\boldsymbol{J}\left(w_{\delta}\right)
$$


Using (35), then adding and subtracting $\theta$ we obtain

$$
\begin{aligned}
\alpha\left\|\boldsymbol{\theta}_{\delta}\right\|_{\Theta}^{2} & \leqslant a_{h}\left(\boldsymbol{\theta}_{\delta}, \boldsymbol{\theta}_{\delta}\right)=a_{h}\left(\boldsymbol{\theta}_{h}-\boldsymbol{\theta}, \boldsymbol{\theta}_{\delta}\right)+a_{h}\left(\boldsymbol{\theta}-\boldsymbol{\theta}_{I}, \boldsymbol{\theta}_{\delta}\right) \\
& =: a_{h}\left(\boldsymbol{\theta}_{h}-\boldsymbol{\theta}, \boldsymbol{\theta}_{\delta}\right)+T_{1} .
\end{aligned}
$$

Then we take $\boldsymbol{\eta}=\boldsymbol{\theta}_{\delta}, v=0$ in (26) and (28), and we add and subtract $\boldsymbol{P}_{h} \boldsymbol{\theta}_{\delta}$, to obtain

$$
\begin{aligned}
a_{h}\left(\boldsymbol{\theta}_{h}-\boldsymbol{\theta}, \boldsymbol{\theta}_{\delta}\right) & =\left(\gamma_{h}, \boldsymbol{P}_{h} \boldsymbol{\theta}_{\delta}\right)-\left(\boldsymbol{\gamma}, \boldsymbol{\theta}_{\delta}\right) \\
= & \left(\gamma_{h}-\gamma, \boldsymbol{P}_{h} \boldsymbol{\theta}_{\delta}\right)+\left(\boldsymbol{\gamma}, \boldsymbol{P}_{h} \boldsymbol{\theta}_{\delta}-\boldsymbol{\theta}_{\delta}\right)=:\left(\gamma_{h}-\gamma, \boldsymbol{P}_{h} \boldsymbol{\theta}_{\delta}\right)+T_{2} .
\end{aligned}
$$

By (47),

$$
\begin{aligned}
\left(\gamma_{h}-\gamma, \boldsymbol{P}_{h} \boldsymbol{\theta}_{\delta}\right) & =-t^{2}\left(\left(\gamma_{h}-\gamma, \gamma_{\delta}\right)+\left(\gamma_{h}-\gamma, \nabla_{h} w_{\delta}-\boldsymbol{J}\left(w_{\delta}\right)\right)\right. \\
& =-t^{2}\left\|\gamma_{\delta}\right\|_{0}^{2}-t^{2}\left(\gamma_{I}-\gamma, \gamma_{\delta}\right)+\left(\gamma_{h}-\gamma, \nabla_{h} w_{\delta}-\boldsymbol{J}\left(w_{\delta}\right)\right) \\
& =:-t^{2}\left\|\gamma_{\delta}\right\|_{0}^{2}+T_{3}+\left(\gamma_{h}-\gamma, \nabla_{h} w_{\delta}-\boldsymbol{J}\left(w_{\delta}\right)\right) .
\end{aligned}
$$

The first term on the right-hand side is negative, and will go to the left in the final estimate. To deal with the last term, we note that (28) with $\boldsymbol{\eta}=0$, $v=w_{\delta}$, and (30) give

$$
\left(\gamma_{h}, \nabla_{h} w_{\delta}-\boldsymbol{J}\left(w_{\delta}\right)\right)=\left(g, w_{\delta}\right)-p_{W}\left(w_{h}, w_{\delta}\right)=\left(g, w_{\delta}\right)+p_{W}\left(w-w_{h}, w_{\delta}\right),
$$

and (26) gives

$$
\left(\gamma, \nabla_{h} w_{\delta}\right)=\left(g, w_{\delta}\right)+j\left(\gamma, w_{\delta}\right)
$$

so

$$
\begin{aligned}
\left(\gamma_{h}-\gamma, \nabla_{h} w_{\delta}-\boldsymbol{J}\left(w_{\delta}\right)\right) & =p_{W}\left(w-w_{h}, w_{\delta}\right)+\left(\gamma, \boldsymbol{J}\left(w_{\delta}\right)\right)-j\left(\gamma, w_{\delta}\right) \\
& =: p_{W}\left(w-w_{h}, w_{\delta}\right)+T_{4} .
\end{aligned}
$$

Finally, adding and subtracting $w_{I}$, and using (42) we deduce

$$
p_{W}\left(w-w_{h}, w_{\delta}\right)=p_{W}\left(w-w_{I}, w_{\delta}\right)-p_{W}\left(w_{\delta}, w_{\delta}\right)=-p_{W}\left(w_{\delta}, w_{\delta}\right) .
$$

The last term on the right-hand side is negative, and goes to the left-hand side. Collecting the above equations we have

$$
\alpha\left\|\boldsymbol{\theta}_{\delta}\right\|_{\Theta}^{2}+t^{2}\left\|\gamma_{\delta}\right\|_{\Gamma}^{2}+p_{W}\left(w_{\delta}, w_{\delta}\right) \leqslant T_{1}+T_{2}+T_{3}+T_{4},
$$


where

$$
\begin{gathered}
T_{1}=a_{h}\left(\boldsymbol{\theta}-\boldsymbol{\theta}_{I}, \boldsymbol{\theta}_{\delta}\right) \leqslant C\left\|\boldsymbol{\theta}-\boldsymbol{\theta}_{I}\right\|_{\Theta}\left\|\boldsymbol{\theta}_{\delta}\right\|_{\Theta}, \\
T_{2}=\left(\boldsymbol{\gamma}, \boldsymbol{P}_{h} \boldsymbol{\theta}_{\delta}-\boldsymbol{\theta}_{\delta}\right), \\
T_{3}=t^{2}\left(\boldsymbol{\gamma}-\gamma_{I}, \gamma_{\delta}\right) \leqslant t^{2}\left\|\gamma-\gamma_{I}\right\|_{0}\left\|\gamma_{\delta}\right\|_{0}, \\
T_{4}=\left(\boldsymbol{\gamma}, \boldsymbol{J}\left(w_{\delta}\right)\right)-j\left(\boldsymbol{\gamma}, w_{\delta}\right) .
\end{gathered}
$$

To estimate $T_{4}$ we add and subtract $\gamma_{I}$ using (30), and then we use (25) and Proposition 4.4, obtaining

$$
\begin{aligned}
T_{4}=\left(\boldsymbol{\gamma}, \boldsymbol{J}\left(w_{\delta}\right)\right)-j\left(\gamma, w_{\delta}\right) & =\left(\boldsymbol{\gamma}-\gamma_{I}, \boldsymbol{J}\left(w_{\delta}\right)\right)-j\left(\gamma-\gamma_{I}, w_{\delta}\right) \\
& \leqslant C\left\|\gamma-\gamma_{I}\right\|_{\Gamma}\left\|w_{\delta}\right\|_{W} .
\end{aligned}
$$

This estimate is not, however, satisfactory, since we do not have a term like $\left\|w_{\delta}\right\|_{W}$ in the left-hand side of (50). Hence, we have to bound $\left\|\nabla_{h} w_{\delta}\right\|_{0}$ as well. For this, we apply (47), Proposition 4.4, and the $L^{2}$-boundedness of $\boldsymbol{P}_{h}$ to obtain

$$
\begin{aligned}
\left\|\nabla_{h} w_{\delta}\right\|_{0} & =\left\|t^{2} \gamma_{\delta}+\boldsymbol{J}\left(w_{\delta}\right)+\boldsymbol{P}_{h} \boldsymbol{\theta}_{\delta}\right\|_{0} \\
& \leqslant C\left(t^{2}\left\|\gamma_{\delta}\right\|_{\Gamma}+\left\|\boldsymbol{\theta}_{\delta}\right\|_{\Theta}+\left(p_{W}\left(w_{\delta}, w_{\delta}\right)\right)^{1 / 2}\right),
\end{aligned}
$$

and therefore, thanks to (22),

$$
\left\|w_{\delta}\right\|_{W} \leqslant C\left(t^{2}\left\|\gamma_{\delta}\right\|_{\Gamma}+\left\|\boldsymbol{\theta}_{\delta}\right\|_{\Theta}+\left(p_{W}\left(w_{\delta}, w_{\delta}\right)\right)^{1 / 2}\right) .
$$

It remains to bound $T_{2}$. From the definition of $\boldsymbol{P}_{h}$, we have

$$
T_{2}=\left(\boldsymbol{\gamma}-\boldsymbol{\delta}, \boldsymbol{P}_{h} \boldsymbol{\theta}_{\delta}-\boldsymbol{\theta}_{\delta}\right) \leqslant\|\boldsymbol{\gamma}-\boldsymbol{\delta}\|_{0}\left\|\boldsymbol{P}_{h} \boldsymbol{\theta}_{\delta}-\boldsymbol{\theta}_{\delta}\right\|_{0} \leqslant C h\|\boldsymbol{\gamma}-\boldsymbol{\delta}\|_{0}\left\|\boldsymbol{\theta}_{\delta}\right\|_{\Theta},
$$

where $\delta$ is an arbitrary element of $\Gamma_{h}^{*}$. We may choose, for example, $\delta$ to be the $L^{2}$-projection of $\gamma$ onto $\underset{k-2}{\sim 0}\left(\mathcal{T}_{h}\right)$ and get $\|\gamma-\delta\|_{0} \leqslant C h^{k-1}\|\gamma\|_{k-1}$. Thus

$$
T_{2} \leqslant C h^{k}\|\gamma\|_{k-1}\left\|\boldsymbol{\theta}_{\delta}\right\|_{\Theta} .
$$

Combining the preceding estimates and invoking the arithmeticgeometric mean inequality we obtain

$$
\begin{aligned}
& \left\|\boldsymbol{\theta}_{\delta}\right\|_{\Theta}^{2}+t^{2}\left\|\gamma_{\delta}\right\|_{\Gamma}+p_{W}\left(w_{\delta}, w_{\delta}\right) \\
& \quad \leqslant C\left(\left\|\boldsymbol{\theta}-\boldsymbol{\theta}_{I}\right\|_{\Theta}^{2}+\left(1+t^{2}\right)\left\|\gamma-\gamma_{I}\right\|_{\Gamma}^{2}+\left\|w-w_{I}\right\|_{W}^{2}+h^{2 k}\|\gamma\|_{k-1}^{2}\right) .
\end{aligned}
$$


In view of (55), this becomes

$$
\begin{aligned}
& \left\|\boldsymbol{\theta}_{\delta}\right\|_{\Theta}^{2}+t^{2}\left\|\gamma_{\delta}\right\|_{\Gamma}+\left\|w_{\delta}\right\|_{W}^{2} \\
& \quad \leqslant C\left(\left\|\boldsymbol{\theta}-\boldsymbol{\theta}_{I}\right\|_{\Theta}^{2}+\left(1+t^{2}\right)\left\|\boldsymbol{\gamma}-\gamma_{I}\right\|_{\Gamma}^{2}+\left\|w-w_{I}\right\|_{W}^{2}+h^{2 k}\|\boldsymbol{\gamma}\|_{k-1}^{2}\right) .
\end{aligned}
$$

Finally, combining with the triangle inequality and the interpolation error bounds (37), (41), (43), and assuming as natural that $t$ is bounded from above, we complete the proof of the Theorem 4.2.

\section{CONTINUOUS $\theta$ AND NONCONFORMING $w$}

In this final section we consider a method in which $\theta$ is discretized by means of continuous elements, and $w$ by means of nonconforming ones. Our method is still of the form (12) and (13), and again we must specify the finite element spaces $\boldsymbol{\Theta}_{h}, W_{h}$, and $\boldsymbol{\Gamma}_{h}$, the penalty functions $p_{\Theta}$ and $p_{W}$, and the projection operator $\boldsymbol{P}_{h}$. The penalty functions are not needed for this method, and can be taken to vanish. We keep $\boldsymbol{\Gamma}_{h}={ }_{k-1}^{v 0}\left(\mathcal{T}_{h}\right)$ as before, and we keep the definition (18) of $\boldsymbol{P}_{h}$ where $\boldsymbol{\Gamma}_{h}^{*}$ is still given by (17). For the choice of $W_{h}$ we take the space of nonconforming piecewise polynomials of degree at most $k$, that is

$$
W_{h}=\left\{v_{h} \in \mathcal{L}_{k}^{0} \mid \boldsymbol{Q}_{e}\left[v_{h}\right]=0, \quad e \in \mathcal{E}_{h}\right\},
$$

where $\boldsymbol{Q}_{e}$ is as before the $L^{2}$-projection on the space of polynomials of degree $k-1$ on $e$. Obviously we still have $\nabla_{h}\left(W_{h}\right) \subset \boldsymbol{\Gamma}_{h}$.

The above definitions allow us to again take $\gamma_{I}:=\boldsymbol{P}_{h} \boldsymbol{\gamma} \in \boldsymbol{\Gamma}_{h}$ and to again define $w_{I} \in W_{h}$ by (38) and (39). Then (40) still holds. In order to have the fundamental property (44), on which the error analysis is based, we need to define the space $\boldsymbol{\Theta}_{h}$ so it admits an interpolation operator $\boldsymbol{\theta} \mapsto \boldsymbol{\theta}_{I} \in \boldsymbol{\Theta}_{h}$ satisfying (36) (which, together with (40), implies (44)). The continuity we are requiring for $\boldsymbol{\theta}_{I}$ precludes the choice $\boldsymbol{\theta}_{I}=\boldsymbol{P}_{h} \boldsymbol{\theta}$ made formerly, and leads to a more complicated construction of $\boldsymbol{\Theta}_{h}$. In particular, the somewhat natural choice $\boldsymbol{\Theta}={ }_{k}^{-1}$ does not work (even for $k=1$ ) as we would not have enough degrees of freedom to force (36) in each element. Instead, we start from ${ }_{k}^{-1}$ and add a sufficient number of bubble functions to ensure (36).

Define $\boldsymbol{\Theta}(T)=\mathcal{P}_{k}(T)+b_{T} \Gamma^{*}(T)$, and remark that all the bubbles of $\mathcal{P}_{k}(T)$ can be written as $b_{T} \mathcal{P}_{k-3}(T)$. Since $\mathcal{P}_{k-3}(T) \subset \Gamma^{*}(T)$, all the bubbles of $\mathcal{P}_{k}(T)$ belong to $b_{T} \Gamma^{*}(T)$. Hence a set of degrees of freedom for $\boldsymbol{\theta} \in \boldsymbol{\Theta}(T)$ consists of the values of each component of $\boldsymbol{\theta}$ at the vertices of $T$, the moments of degree at most $k-2$ of each component of $\boldsymbol{\theta}$ on each 
edge of $T$, and the integrals $\int_{T} \boldsymbol{\theta} \cdot \boldsymbol{\eta} d x$ for each $\boldsymbol{\eta}$ in a basis for $\boldsymbol{\Gamma}^{*}(T)$. Hence, we can set

$$
\boldsymbol{\Theta}_{h}=\left\{\boldsymbol{\theta} \in \boldsymbol{H}_{0}^{1}(\Omega)|\boldsymbol{\theta}|_{T} \in \boldsymbol{\Theta}(T), \quad T \in \mathcal{T}_{h}\right\},
$$

and use the above degrees of freedom to construct a projection operator $\boldsymbol{C}(T) \rightarrow \boldsymbol{\Theta}(T)$, and so an operator $\boldsymbol{\theta} \mapsto \boldsymbol{\theta}_{I}$ from $\boldsymbol{C}(\Omega) \cap \boldsymbol{H}_{0}^{1}(\Omega) \rightarrow \boldsymbol{\Theta}_{h}$. It is then clear that for this operator (36) holds.

Because of the continuity of the elements of $\boldsymbol{\Theta}_{h}$ and the near continuity of the elements of $W_{h}$, all the terms involving edge integrals in (12) and (13) vanish, and the method may be simply written

$$
\begin{gathered}
\left.\left(\mathrm{C} \varepsilon\left(\boldsymbol{\theta}_{h}\right), \varepsilon(\boldsymbol{\eta})\right)+\left(\gamma_{h}, \nabla_{h} v-\boldsymbol{P}_{h} \boldsymbol{\eta}\right)\right)=(g, v), \quad(\boldsymbol{\eta}, v) \in \boldsymbol{\Theta}_{h} \times W_{h}, \\
\left(\nabla_{h} w_{h}-\boldsymbol{P}_{h} \boldsymbol{\theta}_{h}, \boldsymbol{\tau}\right)-t^{2}\left(\gamma_{h}, \boldsymbol{\tau}\right)=0, \quad \boldsymbol{\tau} \in \boldsymbol{\Gamma}_{h} .
\end{gathered}
$$

Remark 5.1. In the lowest order case, $k=1, \boldsymbol{\Gamma}_{h}=\boldsymbol{\Gamma}_{h}^{*}$ is just the space of piecewise constants and $\boldsymbol{P}_{h}$ the $L^{2}$-projection into this space, $\boldsymbol{\Theta}_{h}$ is the usual space of conforming piecewise linears augmented by bubbles, and $W_{h}$ the usual space on nonconforming piecewise linears, so this method is exactly that of Arnold and Falk [5].

Remark 5.2. The choice of $\boldsymbol{\Theta}_{h}$ was made in order to obtain (36) easily, rather than to simplify the implementation of the method. From the latter point of view, the alternative choice based on $\Theta(T):=\mathcal{P}_{k}(T)+$ $b_{T} \mathcal{P}_{k-1}$ (which coincides with our choice only for $k=1$ ) seems natural, but we shall not consider this possibility here.

Equation (58) may be written in strong form as

$$
t^{2} \gamma_{h}=\nabla_{h} w_{h}-\boldsymbol{P}_{h} \boldsymbol{\theta}_{h}
$$

which makes it easy to see that the method admits a unique solution. Moreover, (59) and (44) together give

$$
t^{2} \gamma_{\delta}=\nabla_{h} w_{\delta}-\boldsymbol{P}_{h} \boldsymbol{\theta}_{\delta}
$$

(with the notation given by (46)). We now turn to the error analysis, assuming that $k \geqslant 3$ (since the case $k=1$ is handled in [5]).

The analysis proceeds along the same lines as in the previous section. Following (48) and (49) we obtain

$$
\alpha\left\|\boldsymbol{\theta}_{\delta}\right\|_{\Theta}^{2} \leqslant T_{1}+T_{2}+\left(\gamma_{h}-\gamma, P_{h} \boldsymbol{\theta}_{\delta}\right)
$$


with $T_{1}$ and $T_{2}$ still given by (51) and (52). We can now use (60), add and subtract $\gamma_{I}$, use (57) and (10) (both with $\boldsymbol{\eta}=0$ ), and the definition of $j$ given by (23) to obtain:

$$
\begin{aligned}
\left(\gamma_{h}-\gamma, P_{h} \boldsymbol{\theta}_{\delta}\right) & =-t^{2}\left(\gamma_{h}-\gamma, \gamma_{\delta}\right)+\left(\gamma_{h}-\gamma, \nabla_{h} w_{\delta}\right) \\
& =-t^{2}\left\|\gamma_{\delta}\right\|_{0}^{2}-t^{2}\left(\gamma_{I}-\gamma, \gamma_{\delta}\right)+\left(\gamma_{h}-\gamma, \nabla_{h} w_{\delta}\right) \\
& =:-t^{2}\left\|\gamma_{\delta}\right\|_{0}^{2}+T_{3}+\left(\gamma_{h}-\gamma, \nabla_{h} w_{\delta}\right) \\
& =-t^{2}\left\|\gamma_{\delta}\right\|_{0}^{2}+T_{3}-j\left(\gamma, w_{\delta}\right),
\end{aligned}
$$

where the first term on the right-hand side is negative, and will go to the left in the final estimate, and $T_{3}$ is given by (53). It remains to bound the last term.

Let $\gamma_{M}$ denote the BDM interpolant of $\gamma$ of degree $k-1$ (see, e.g. [13] or [14]). Thus $\gamma_{M} \in{ }_{k-1}^{\sim 0}\left(\mathcal{T}_{h}\right)$ satisfies: (i) its normal component is continuous across interelement boundaries, (ii) $\operatorname{div} \gamma_{M}=P_{k-2} \operatorname{div} \gamma=P_{k-2} g$, where $P_{k-2}$ denotes the $L^{2}$-projection onto ${ }_{k-2}\left(\mathcal{T}_{h}\right)$, and (iii) $\gamma-\gamma_{M}$ is orthogonal to $\stackrel{\sim 0}{0}$.

Using the definition (23) of $j(\cdot, \cdot)$, then (56), then (2) and Green formula in each $T$, then (60) and (ii), then (iii) and (ii), then CauchySchwarz, the arithmetic-geometric mean inequality and finally standard interpolation estimates, we get

$$
\begin{aligned}
j\left(\boldsymbol{\gamma}, w_{\delta}\right)= & j\left(\boldsymbol{\gamma}-\gamma_{M}, w_{\delta}\right) \\
= & \left(\boldsymbol{\gamma}-\gamma_{M}, \nabla_{h} w_{\delta}\right)+\left(\operatorname{div} \gamma-\operatorname{div} \gamma_{M}, w_{\delta}\right) \\
= & \left(\boldsymbol{\gamma}-\gamma_{M}, t^{2} \gamma_{\delta}+\boldsymbol{P}_{h} \boldsymbol{\theta}_{\delta}\right)+\left(g-P_{k-2} g, w_{\delta}\right) \\
= & t^{2}\left(\boldsymbol{\gamma}-\gamma_{M}, \gamma_{\delta}\right)+\left(\boldsymbol{\gamma}-\gamma_{M},\left(I-P_{0}\right) \boldsymbol{P}_{h} \boldsymbol{\theta}_{\delta}\right) \\
& \quad+\left(g-P_{k-2} g,\left(I-P_{0}\right) w_{\delta}\right) \\
\leqslant & \frac{1}{2 \varepsilon} t^{2}\left\|\gamma-\gamma_{M}\right\|_{0}^{2}+\frac{\varepsilon}{2} t^{2}\left\|\gamma_{\delta}\right\|_{0}^{2}+\left\|\gamma-\gamma_{M}\right\|_{0}\left\|\left(I-P_{0}\right) \boldsymbol{P}_{h} \boldsymbol{\theta}_{\delta}\right\|_{0} \\
& \quad+\left\|g-P_{k-2} g\right\|_{0}\left\|\left(I-P_{0}\right) w_{\delta}\right\|_{0} \\
\leqslant & \frac{1}{2 \varepsilon} t^{2} h^{2 k}\|\gamma\|_{k}^{2}+\frac{\varepsilon}{2} t^{2}\left\|\gamma_{\delta}\right\|_{0}^{2}+C h^{k-1}\|\gamma\|_{k-1} h\left\|\boldsymbol{\theta}_{\delta}\right\| \\
& \quad+C h^{k-1}\|g\|_{k-1} h\left\|w_{\delta}\right\| \|_{W} .
\end{aligned}
$$

The remainder of the error analysis follows the lines of the previous section, arriving finally to the error bound

$$
\begin{aligned}
& \left\|\boldsymbol{\theta}-\boldsymbol{\theta}_{h}\right\|_{\Theta}+\left\|w-w_{h}\right\|_{W}+t\left\|\boldsymbol{\gamma}-\gamma_{h}\right\|_{\Gamma} \\
& \quad \leqslant C h^{k}\left(\|\boldsymbol{\theta}\|_{k+1, \Omega}+\|w\|_{k+1, \Omega}+t\|\gamma\|_{k}+\|\gamma\|_{k-1}+\|g\|_{k-1}\right) .
\end{aligned}
$$




\section{ACKNOWLEDGMENTS}

D. N. Arnold is supported by NSF Grant DMS-0107233 and F. Brezzi and L. D. Marini are partially supported by Italian government Grant PRIN 2001.

\section{REFERENCES}

1. Agmon, S. (1965). Lectures on Elliptic Boundary Value Problems, Van Nostrand Mathematical Studies, Princeton, NJ.

2. Arnold, D. N. (1982). An interior penalty finite element method with discontinuous elements. SIAM J. Numer. Anal. 19, 742-760.

3. Arnold, D. N., Brezzi, F., Cockburn, B., and Marini, L. D. (2000). Discontinuous Galerkin methods for elliptic problems. In Discontinuous Galerkin Methods (Newport, RI, 1999), Lecture Notes Computational Science Engineering Vol. 11, Springer, Berlin, pp. 89101.

4. Arnold, D. N., Brezzi, F., Cockburn, B., and Marini, L. D. (2002). Unified analysis of discontinuous Galerkin methods for elliptic problems. SIAM J. Numer. Anal. 39, 17491779 .

5. Arnold, D. N., and Falk, R. S. (1989). A uniformly accurate finite element method for the Reissner-Mindlin plate. SIAM J. Numer. Anal. 26, 1276-1290.

6. Auricchio, F., and Lovadina, C. (1999). Partial selective reduced integration schemes and kinematically linked interpolations for plate bending problems. Math. Models Meth. Appl. Sci. 9, 693-722.

7. Auricchio, F., and Lovadina, C. (2001). Analysis of kinematic linked interpolation methods for Reissner-Mindlin plate problems. Comput. Meth. Appl. Mech. Eng. 190, 18-19.

8. Auricchio, F., and Taylor, R. L. (1994). A shear deformable plate element with an exact thin limit. Comput. Meth. Appl. Mech. Eng. 118, 393-412.

9. Bathe, K. J. (1994). Finite Element Procedures, Prentice-Hall, Englewood Cliffs, NJ.

10. Brenner, S. C. (2003). Poincaré-Friedrichs inequalities for piecewise $H^{1}$ functions. SIAM J. Numer. Anal. 41, 306-324.

11. Brenner, S. C. (2004). Korn's inequalities for piecewise $H^{1}$ vector fields. Math. Comput. 73, 1067-1087.

12. Brezzi, F., Bathe, K. J., and Fortin, M. (1989). Mixed-interpolated elements for ReissnerMindlin plates. Int. J. Numer. Meth. Eng. 28, 1787-1801.

13. Brezzi, F., Douglas, J. Jr., and Marini, L. D. (1985). Two families of mixed finite elements for second order elliptic problems. Numer. Math. 47, 217-235.

14. Brezzi, F., and Fortin, M. (1999). Mixed and Hybrid Finite Element Methods, Springer, New York.

15. Brezzi, F., Fortin, M., and Stenberg, R. (1991). Error analysis of mixed-interpolated elements for Reissner-Mindlin plates. Math. Models Meth. Appl. Sci. 1, 125-151.

16. Brezzi, F., and Marini, L. D. (2003). A nonconforming element for the Reissner-Mindlin plate. Comput. Struct. 81, 515-522.

17. Bucalem, M. L., and Bathe, K. J. (1997). Finite element analysis of shell structures. Arch. Comput. Meth. Eng. 4, 3-61.

18. Bucalem, M. L., and Bathe, K. J. (1993). Higher-order MITC general shell elements. Int. J. Numer. Meth. Eng. 36, 3729-3754.

19. Chapelle, D., and Bathe, K. J. (1998). Fundamental considerations for the finite element analysis of shell structures. Comput. Struct. 66, 19-36. 
20. Chapelle, D., and R. Stenberg, R. (1999). Stabilized finite element formulations for shells in a bending dominated state. SIAM J. Numer. Anal. 36, 32-73.

21. Duran, R., and Liberman, E. (1992). On mixed finite-element methods for the ReissnerMindlin plate model. Math. Comput. 58, 561-573.

22. Flores, F. G., Oñate, E., and Zaráte, F. (1995). New assumed strain triangles for nonlinear shell analysis. Comput. Mech. 17, 107-114.

23. Hansbo, P., and Larson, M. G. (2000). Discontinuous Galerkin and the CrouzeixRaviart element: Application to Elasticity, Chalmers Finite Element Center Preprint 2000-9, Chalmers University of Technology, Sweden.

24. Hughes, T. J. R. (1987). The Finite Element Method: Linear Static and Dynamic Finite Element Analysis, Prentice-Hall, Englewood Cliffs, NJ.

25. Lovadina, C. (1996). A new class of mixed finite element methods for Reissner-Mindlin plates. SIAM J. Numer. Anal. 33, 2457-2467.

26. Lovadina, C. (1998). Analysis of a mixed finite element method for the Reissner-Mindlin plate problems. Comput. Meth. Appl. Mech. Eng. 163, 71-85.

27. Ming, P.-B., and Shi, Z.-C. (2001). Nonconforming rotated $Q_{1}$ element for ReissnerMindlin plate. Math. Models Meth. Appl. Sci. 11, 1311-1342. 